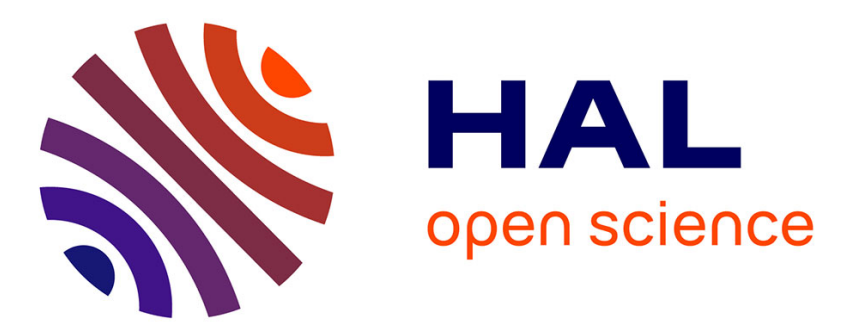

\title{
Task-based whole-body control of humanoid robots with ZMP regulation, real-time application to a squat-like motion
}

David Galdeano, Ahmed Chemori, Sébastien Krut, Philippe Fraisse

\section{- To cite this version:}

David Galdeano, Ahmed Chemori, Sébastien Krut, Philippe Fraisse. Task-based whole-body control of humanoid robots with ZMP regulation, real-time application to a squat-like motion. SSD: Systems, Signals and Devices, Feb 2014, Castelldefels-Barcelona, Spain. 10.1109/SSD.2014.6808778 . lirmm00945690

\author{
HAL Id: lirmm-00945690 \\ https://hal-lirmm.ccsd.cnrs.fr/lirmm-00945690
}

Submitted on 12 Feb 2014

HAL is a multi-disciplinary open access archive for the deposit and dissemination of scientific research documents, whether they are published or not. The documents may come from teaching and research institutions in France or abroad, or from public or private research centers.
L'archive ouverte pluridisciplinaire HAL, est destinée au dépôt et à la diffusion de documents scientifiques de niveau recherche, publiés ou non, émanant des établissements d'enseignement et de recherche français ou étrangers, des laboratoires publics ou privés. 


\title{
Task-based whole-body control of humanoid robots with ZMP regulation, real-time application to a squat-like motion
}

\author{
David Galdeano, Ahmed Chemori, Sébastien Krut and Philippe Fraisse \\ LIRMM, Université Montpellier 2, \\ LIRMM - UMR 5506 - CC 477, 161 rue Ada, \\ 34095 Montpellier Cedex 5, France \\ Email: galdeano(chemori,krut,fraisse)@lirmm.fr
}

\begin{abstract}
In this paper, a task based whole-body control strategy is proposed for humanoid robots. Its basic idea lies in the control of (i) the CoM with a ZMP regulation, (ii) the relative pose of robot's feet and (iii) joint's limit avoidance. Through the proposed study, it is shown that these tasks allow to produce smooth whole-body motions. Real-time experimentation results are presented to show the effectiveness of the proposed control scheme.
\end{abstract}

\section{INTRODUCTION}

Since the early days of the humanoid robotics, most of studies has been focused on two field: (i) the study of biped walking control and stability issues targeting the lower-limbs considering the upper-limbs as a dummy mass [1], (ii) the manipulation of items or other tasks involving upper-limbs motions only without making a step [2].

This separation has allowed a simpler study of humanoid control but trying to combine upper and lower-limbs motion together is not an easy process if these are designed separately since stability is not ensured [3]. To tackle this issue and improve the efficiency of control, the humanoid robot has to be considered as a whole system.

In the literature, several approaches have been proposed to deal with whole-body control; most of them can be classified into three classes: optimization based control, human-capture based control and task-based control.

Optimization based control relies on accurate model of the robot and his environment to compute off-line the best joints' trajectories. Several studies [4], [5] use this methodology to produce whole-body motions. However, this method has several drawbacks: (i) some optimization can take a very long time to compute [6], (ii) accurate model of the environment are difficult to produce and (iii) this approach is not reactive hence difficult to use in a dynamic environment.

Human capture based control relies in using sensors to record human motion data. Then these last ones are used to generate human-like motions such as balancing [7], walking [8], or dancing [7], [8]. However, despite evident similarities, the human locomotor system is much more complex than the humanoid's one. Therefore, a direct mapping from human captured motion to humanoid robot will fail in most cases and often lead to instable motions. This implies that data need to be adapted to take into account the specific structures of humanoid robots such as joint limits [9].
Task-based control uses the principle of task as defined by Nakamura [10] and Siciliano [11] to track different objectives in operational space using a joint space control. Applications to humanoid robot can be found in Mansard, Ramos and Sentis works [12], [13], [14]. It is worth to note that Montecillo [7] had based his work on both human capture data and tasks hierarchy controls.

In this paper, a whole-body control strategy is proposed. The basic idea of the proposed control strategy is to use the position of the Center of Mass (CoM) and the relative pose of the feet to produce stable motions. By using Siciliano's recursive formulation of task formalism [11], we can track these two quantities. We added (i) a zero moment point (ZMP) stabilizer to improve the stability of the robot and (ii) a joints' limit avoidance to deal with singularities issue.

Every approach proposed in the literature make a decomposition of walking motion in different phases. The usual ones are simple support, double support and impact phases. Our approach allows to avoid this phenomenon. We can create a continuous whole-body control without having to switch from phases like double support to single support when a foot is lifted. The dynamic stability is ensured by the ZMP regulator.

This paper is organized as follows: in next section, our demonstrator HOAP-3 is introduced. Section III is devoted to the proposed control scheme, where its basic principle is presented and discussed. Real-time experimental results are presented in section IV, with a presentation and discussion of the obtained results. The paper ends with some concluding remarks and future work.

\section{DESCRIPTION AND MODELING OF HOAP-3}

In this study, we propose to use the humanoid robot HOAP3 from Fujitsu company as a testbed for the illustration of the proposed control solution (cf. Fig. 1-(a)).

This robot is a small humanoid of $60 \mathrm{~cm}$ tall, $8.8 \mathrm{~kg}$ weight, and has 28 degrees of freedom (dof) distributed as follows: Each leg is composed of six dof, three dof at the hip, one dof at the knee and two dof at the ankle. The torso is composed of 1 dof and the neck is composed of 3 dof. Both arms are composed of 6 dof, 3 dof at the shoulder, one dof at the elbow and two dof at the hand. The spatial distribution of the different dofs is illustrated in Fig. 1-(b). 


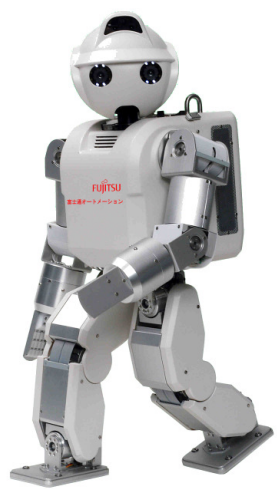

(a) The HOAP-3 robot

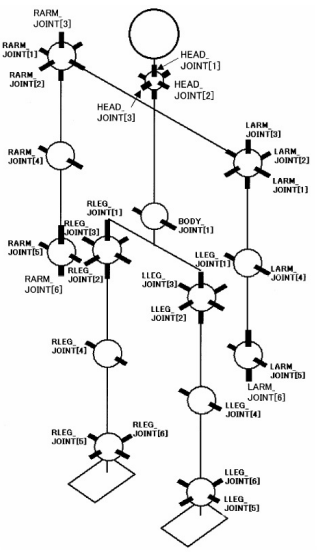

(b) HOAP-3 kinematics model
Fig. 1: The HOAP-3 humanoid robot used as demonstrator for real-time experiments.

The robot is equipped with optical incremental encoders at all joints, a 3-axis accelerometer and 3-axis gyroscope used for posture sensing, four force sensors per foot for ground contact forces' measurement and two cameras for visual feedback.

The actuators are brushless motors paired with microcontrollers, which are controlled by an embedded PC running with a real time kernel RT-Linux at a sample frequency of 1 $\mathrm{kHz}$.

\section{PROPOSED CONTROL SCHEME}

As previously mentioned in the introduction, the challenge to perform a whole-body control law is still an open problem. The use of task formalism to control a robot for tracking several objectives in the operational space has been proposed by Nakamura in [10] and Siciliano in [11]. The task formalism has been used on humanoid robots by Sentis and Khatib in [14] for multi-contact dynamic motions. Recently, Mansard [13], [15] has used this approach on humanoid robots and has generalized the formalism by using the addition and removal of tasks during the control execution. He has also provide a flexible $\mathrm{C}++$ implementation of the proposed algorithm.

The actual study is also based on the task formalism. The main contributions of this paper is a whole-body control architecture including a ZMP regulation to ensure stability. A first study has been proposed in [16] to deal with whole-body control, but without any experimental results.

In the literature, several tasks are needed to produce stable whole-body motions. However, in this work, the proposed architecture is focused on only three main tasks: the relative feet position and orientation tracking, CoM position tracking with ZMP regulation and the joints' limits avoidance.

It is worth to note that additional tasks such as manipulation or Human-Robot Interaction (HRI) tasks can easily be added within this framework.

\section{A. First task: Relative feet pose}

The relative feet pose task allows to place a foot relatively to the other one (cf. Fig. 2).

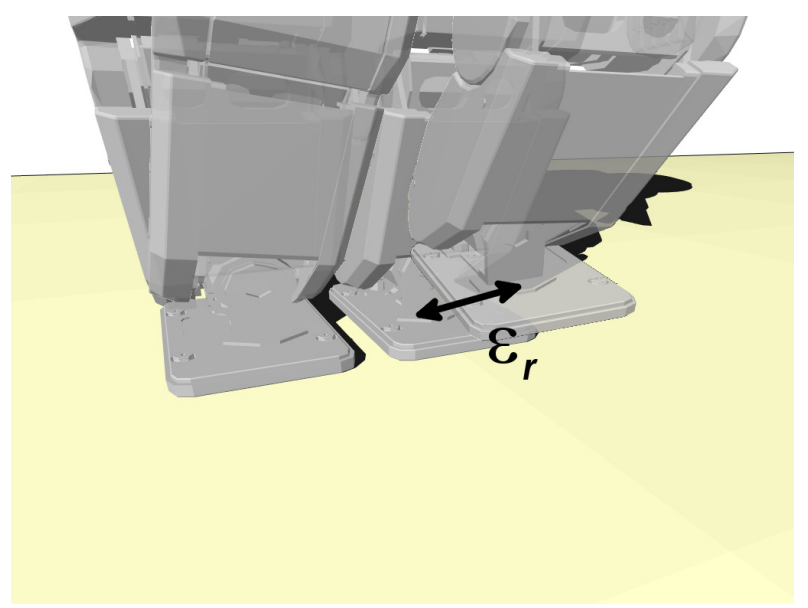

Fig. 2: Illustration of the tracking error on the relative feet pose.

It is based on the tracking error of the relative feet pose:

$$
\varepsilon_{r}=\left[\begin{array}{ll}
E_{\text {pos }}^{T} & E_{o r i}^{T}
\end{array}\right]^{T}
$$

where $E_{\text {pos }} \in \mathbb{R}^{3 \times 1}$ is the position error and $E_{\text {or } i} \in \mathbb{R}^{3 \times 1}$ is the orientation error.

The position error is defined by:

$$
E_{\text {pos }}=P_{r d}-P_{r}
$$

where $P_{r d}=\left[\begin{array}{lll}x_{r d} & y_{r d} & z_{r d}\end{array}\right]^{T}$ expresses the desired feet relative position, designed to produce a stable motion and $P_{r}=P_{r f}-P_{l f}$ is the estimated feet relative position where $P_{r f}=\left[\begin{array}{lll}x_{r f} & y_{r f} & z_{r f}\end{array}\right]^{T}$ and $P_{l f}=\left[\begin{array}{lll}x_{l f} & y_{l f} & z_{l f}\end{array}\right]^{T}$ are respectively the positions of the right and left foot obtained using the forward kinematic model $\left(F K M_{r}\right)$.

The orientation error is defined by:

$$
E_{\text {ori }}=R_{r f}\left(\ln \left(R_{r f}^{-1} R_{l f} R_{r d}\right)\right)^{\vee}
$$

where $R_{r d} \in \mathbb{R}^{3 \times 3}$ is the desired feet relative orientation matrix, $R_{r f} \in \mathbb{R}^{3 \times 3}$ and $R_{l f} \in \mathbb{R}^{3 \times 3}$ are respectively the right and left foot orientation matrix obtained using $F K M_{r}$.

The logarithmic map operator $\omega=(\ln R)^{\vee}$ is defined by:

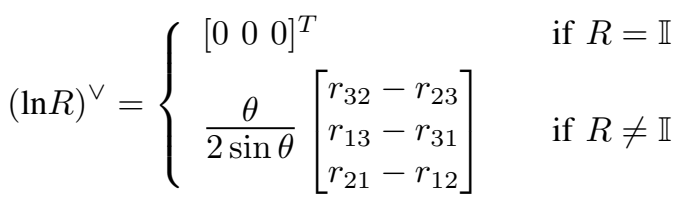

with:

$R=\left[\begin{array}{lll}r_{11} & r_{12} & r_{13} \\ r_{21} & r_{22} & r_{23} \\ r_{31} & r_{32} & r_{33}\end{array}\right]$ and $\theta=\cos ^{-1}\left(\frac{r_{11}+r_{22}+r_{33}-1}{2}\right)$

where $\mathbb{I} \in \mathbb{R}^{3 \times 3}$ is the identity matrix. More details on this operator can be found in [17] and [18]. 
The orientation error is computed form the rotation matrix $\left(R \in \mathbb{R}^{3 \times 3}\right.$ ) to have a well defined error without gimbal lock limitations which appears with Euler angles notation. These orientation matrix needs only to be defined in a common reference coordinates. Here, we take the reference foot orientation as a common reference coordinates.

The Jacobian of the relative feet pose task is then defined as follows:

$$
\varepsilon_{r}=J_{r} \varepsilon_{q}
$$

where $\varepsilon_{q} \in \mathbb{R}^{28 \times 1}$ represents the vector of articular position errors of the robot, $J_{r} \in \mathbb{R}^{6 \times 28}$ is the Jacobian matrix of the relative translation between the feet.

\section{B. Second task: Center of mass position}

The Center of Mass (CoM) position tracking allows to improve the stability in the static case. This is a common task in the task formalism applied to humanoid robots [7], [19], [20] since it is a usually used stability indicator. By tracking a CoM designed to achieve stability, we can produce stable motions.

The tracking error of the CoM is expressed by:

$$
\varepsilon_{C o M}=C o M_{d}-C o M
$$

where $C o M_{d}=\left[x_{C o M_{d}} y_{C o M_{d}} z_{C o M_{d}}\right]^{T}$ is the desired position of the center of mass and CoM $=\left[\begin{array}{lll}x_{C o M} & y_{C o M} & z_{C o M}\end{array}\right]^{T}$ is the real position of the center of mass obtained from the forward kinematic model of center of mass $\left(F K M_{C o M}\right)$.

Both of these two positions are expressed in the reference foot coordinates. This allows to design a cyclic trajectory for walking without having to take care about the position of the robot in the global coordinate system. The position of the robot in the global coordinate system can be estimated using odometry method based on foot position.

The jacobian of the CoM task is then defined as follows:

$$
\varepsilon_{C o M}=J_{C o M} \varepsilon_{q}
$$

where $J_{C o M} \in \mathbb{R}^{3 \times 28}$ is the CoM Jacobian matrix.

\section{ZMP regulation}

Now, to improve dynamic stability of the robot, we propose to consider a ZMP regulation. This regulation is obtained by injecting the ZMP error in the CoM tracking task using a Proportional Derivative (PD) controller.

Let us defined the tracking error on the ZMP position, $\varepsilon_{Z M P}$, as follows:

$$
\begin{gathered}
\varepsilon_{Z M P}=\alpha d Z M P_{\text {left }}+(1-\alpha) d Z M P_{\text {right }} \\
\alpha=\frac{A Z M P_{\text {left }}}{A Z M P_{\text {left }}+A Z M P_{\text {right }}} \\
d Z M P_{\text {left }}=Z M P_{\text {left_desired }}-Z M P_{\text {left_measured }} \\
d Z M P_{\text {right }}=Z M P P_{\text {right_desired }}-Z M P_{\text {right_measured }}
\end{gathered}
$$

where $d Z M P_{\text {left }}$ and $d Z M P_{\text {right }}$ are illustrated in Fig. 3. $A Z M P_{\text {left }}$ and $A Z M P_{\text {right }}$ are the amplitudes of forces

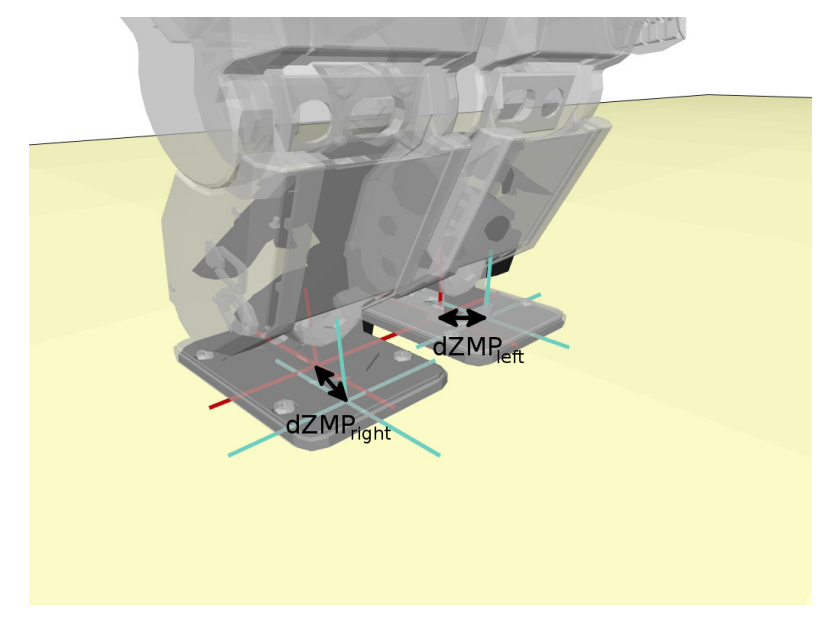

Fig. 3: Graphical illustration of tracking errors on ZMP position.

applied respectively on the right and left soles collected by using force sensors. ZMPleft_desired $\in \mathbb{R}^{2 \times 1}$ and $Z M P_{\text {right_desired }} \in \mathbb{R}^{2 \times 1}$ are the desired ZMP positions respectively under the right and left soles. Theses values are kept constant at the center of each foot $\left(x_{-} Z M P_{\text {desired }}=\right.$ $\left.0 ; y_{-} Z M P_{\text {desired }}=0\right)$. $Z M P_{\text {left_measured }} \in \mathbb{R}^{2 \times 1}$ and $Z M P_{\text {right_measured }} \in \mathbb{R}^{2 \times 1}$ are the actual ZMP position respectively under the right and left sole measured using the force sensors.

In equation (9), the ZMP position tracking error $\varepsilon_{Z M P}$ is a weighted distribution of the ZMP error on both feet. The weight for this distribution is based on the repartition of force amplitude on both feet as defined in equation (10). This allows to neglect the error on one foot when it is lifted off for walking scenarios (for instance) and reinforce the stabilization on the support foot.

The tracking error of the ZMP: $\varepsilon_{Z M P}$, is then injected in the CoM task using a Proportional Derivative (PD) controller.

$$
\varepsilon_{C o M \& Z M P}=\varepsilon_{C o M}+K_{p} \varepsilon_{Z M P}+K_{d} \frac{d \varepsilon_{Z M P}}{d t}
$$

The effectiveness of this part of the controller is demonstrated in section IV-A.

\section{Third task: Joints' limits avoidance}

Using only the two defined previous tasks control can lead the robot to non desired behavior because of drift on joint positions while tracking perfectly these tasks. If the drift leads the robot joints to a singular pose or to joints' articular limits, the induced loss of dof can alter the efficiency of tasks tracking. To overcome this problem, it will be necessary to add some constraints on the control.

The proposed solution to tackle this problem is based on attractive potential fields who allow to defined a comfort position (cf. Fig. 4).

The attractive potential fields are defined as:

$$
\varepsilon_{J o i n t s}=\beta\left[\begin{array}{lllll}
\varepsilon_{q_{1}} & \varepsilon_{q_{2}} & \varepsilon_{q_{3}} & \ldots & \varepsilon_{q_{22}}
\end{array}\right]^{T}
$$




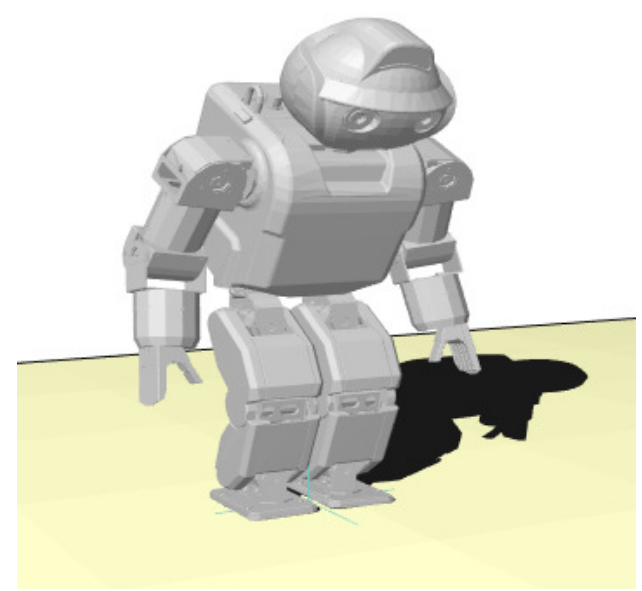

Fig. 4: Robot's comfort position is defined with joints' configuration far from their limits.

with:

$$
\varepsilon_{q_{i}}=\frac{2\left(q_{i}-q_{i m e d}\right)}{\left(q_{\text {imax }}-q_{\text {imin }}\right)^{2}} \quad ; \quad q_{\text {imed }}=\frac{q_{\text {imax }}+q_{i m i n}}{2}
$$

where $\beta \in \mathbb{R}_{+}^{*}$ is a convergence gain, $q_{\text {imax }}$ and $q_{\text {imin }}$ are respectively the upper and lower limit on the joint $i, q_{i}$ is the current joint $i$ position and $q_{\text {imed }}$ is its comfort position.

This specific task must be added after all operational space tasks because it affect all the degrees of freedom. The null space used to add tasks is filled meaning no other task can be tracked.

\section{E. Architecture of the control scheme}

In order to control the robot, we have designed the control scheme summarized in Fig. 5. The task computation algorithm is based on Siciliano's recursive formulation detailed in [11]

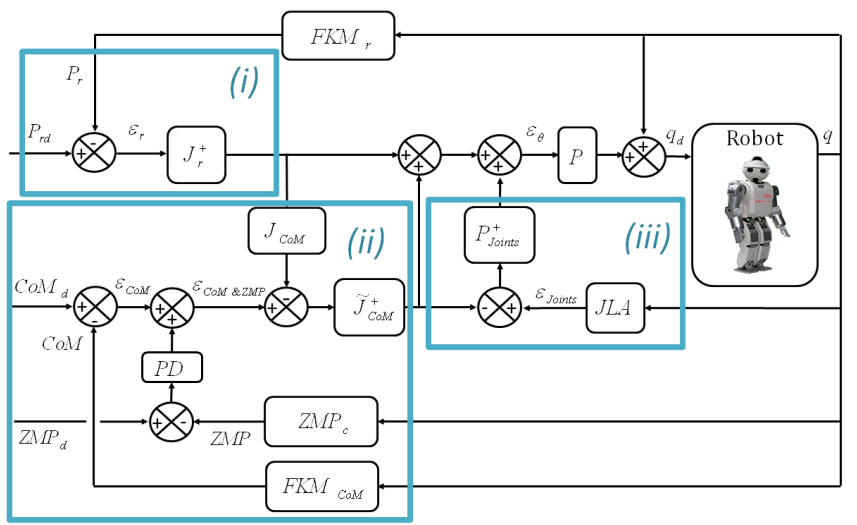

Fig. 5: Block diagram of the proposed control scheme with the control of $(i)$ the relative pose of robot's feet, (ii) the CoM with a ZMP regulation and (iii) joint's limits avoidance.

The feet relative pose is the task with the highest priority since an error in foot placement often lead to fall of the robot. The control law $\varepsilon_{q 1}$ with this task only is given by the following:

$$
\varepsilon_{q 1}=J_{r}^{+} \varepsilon_{r}
$$

The CoM task with ZMP regulation has the second priority since small error on the CoM position are tolerable. Indeed, these errors can often be corrected without a loss of equilibrium. The control with the two tasks becomes $\varepsilon_{q 2}$ given by:

$$
\varepsilon_{q 2}=\varepsilon_{q 1}+\left(\widetilde{J}_{C o M}\right)^{+}\left(\varepsilon_{C o M}-J_{C o M} \varepsilon_{q 1}\right)
$$

with:

$$
\widetilde{J}_{C o M}=J_{C o M} P_{r} \quad \text { and } \quad P_{r}=\left(\mathbb{I}-J_{r}^{+} J_{r}\right)
$$

Other tasks like items manipulations and upper body position and/or orientation can be added now without compromising the motion's stability using Siciliano's recursive formulation [11].

The last task to be considered is the joints' limits avoidance because it provides a good way to avoid the loss of dof due to singularity. The main inconvenient is that the control's nullspace is filled by this task, meaning that no other task can be added after this one. The final control law becomes the following:

$$
\varepsilon_{q 3}=\varepsilon_{q 2}+\left(P_{\text {Joints }}\right)^{+}\left(\varepsilon_{\text {Joints }}-\varepsilon_{q 2}\right)
$$

with:

$$
P_{\text {Joints }}=\left(P_{r}-\widetilde{J}_{\text {CoM }}^{+} \widetilde{J}_{C o M}\right)
$$

This final control law allows to create continuous wholebody motions while ensuring dynamic stability, without having to switch between phases like double support and single support in dynamic walking tasks.

\section{REAL-TIME EXPERIMENTAL RESULTS}

The proposed control scheme (cf. Fig. 5) has been developed and implemented in the HOAP-3 robot (presented in section II) with reference signals obtained by numerical computation. These reference signals depends on the two scenarios to be performed.

In the first one, the reference signals given for the relative feet pose are constant, which means that we want the feet to be static, and the reference CoM position is set to the initial robot's CoM position and kept constant which means that the robot should not move excepted for ZMP regulation.

In the second one, the reference signals given for the relative feet pose are constant, which means that we want the feet to be also static, and the reference CoM position to be moved up and down using a sinus signal to generate a squat-like motion. The ZMP regulation ensure that the produced motions are stable. 


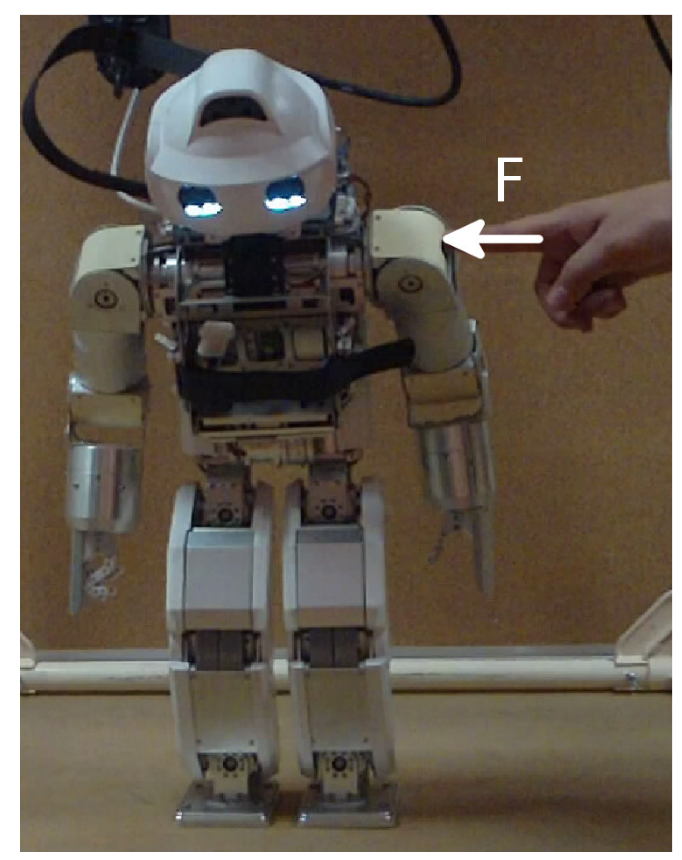

Fig. 6: Static standing under disturbing forces.

\section{A. First scenario: static standing under disturbing forces}

The objective of this scenario is to prove the effectiveness of the ZMP regulation presented in section III-C.

We deliberately push the robot while controlling the wholebody to keep constant feet relative pose and a fixed CoM (cf. Fig. 6).

The only motion from the robot is due to the $\mathrm{ZMP}$ regulation inside CoM task.

The ZMP measurement resulting from this scenario are displayed in Fig. 7.

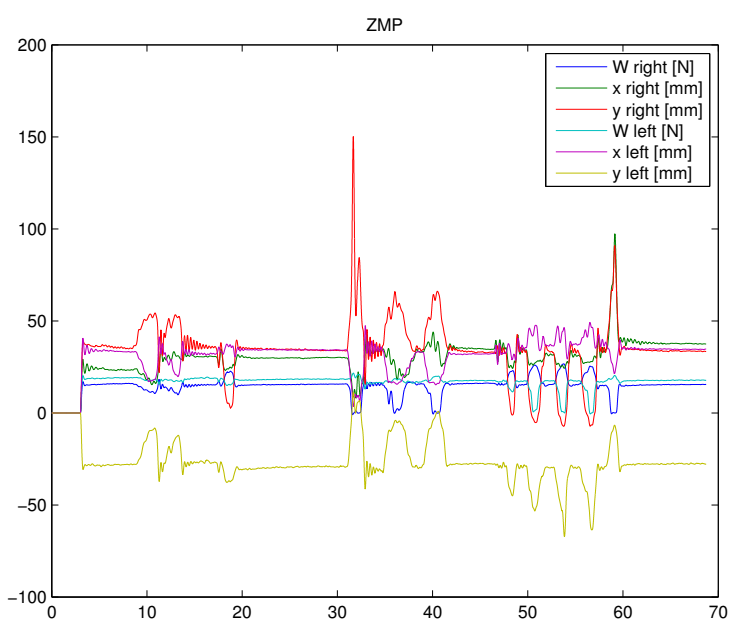

Fig. 7: ZMP measurements.

In Fig. 7, $W$ left and $W$ right are the amplitudes of the measured forces for respectively the left and right foot. $\mathrm{x}$ and $\mathrm{y}$ are respectively the frontal and lateral positions of the ZMP relative to the center of each foot.

It can clearly be observed that the robot reaction to pushing allows it to keep a dynamic stability. When the robot is pushed, it resists to the applied external force and commes back quickly to the desired ZMP position with little oscillations as soon as the disturbance vanishes.

\section{B. Second scenario: squat-like motions}

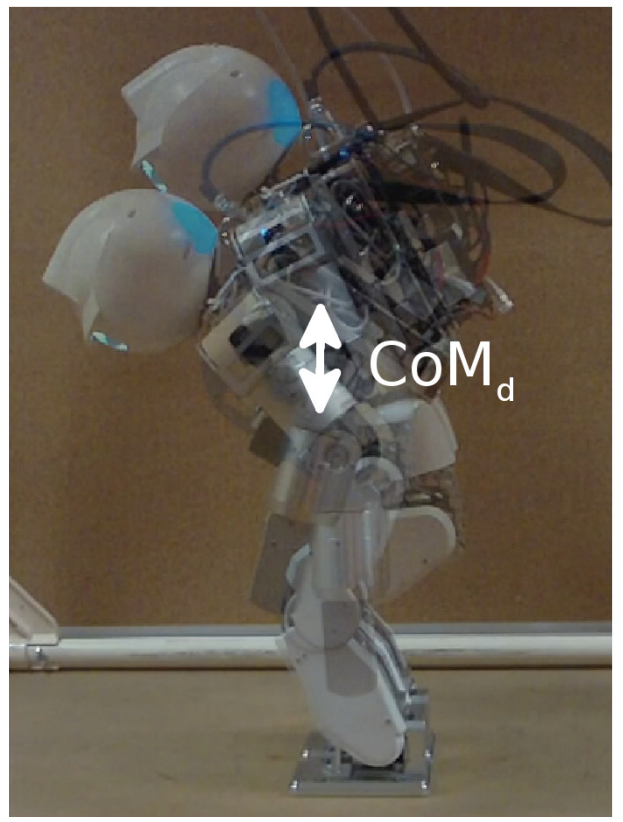

Fig. 8: Squat-like motions.

The objective of this scenario is to produce a whole-body control of the HOAP-3 by moving the CoM position up and down according to a sinusoidal signal (cf. Fig. 8).

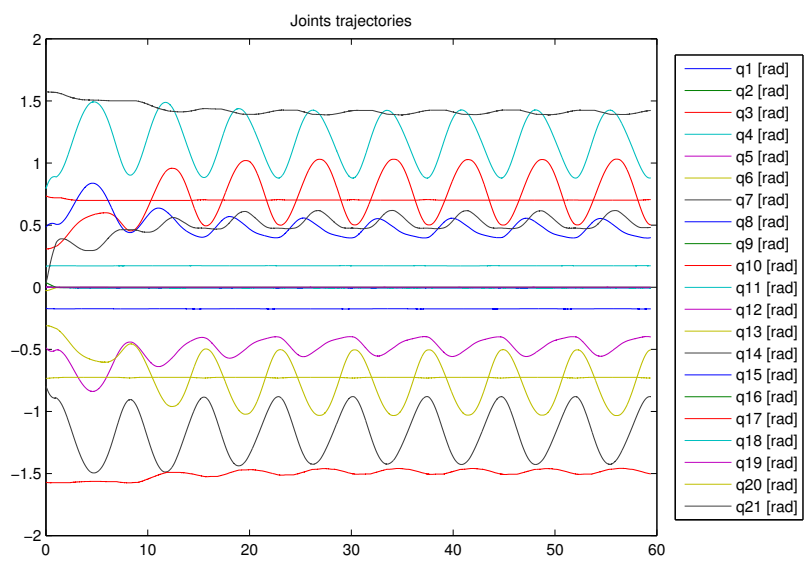

Fig. 9: Joints trajectories.

In Fig. 9, the joints' trajectories are displayed. It is worth to note that the trajectories converge to a periodic motion since 
the desired tasks are periodic, some joints are not solicited since not all of them are useful for this whole-body motion.

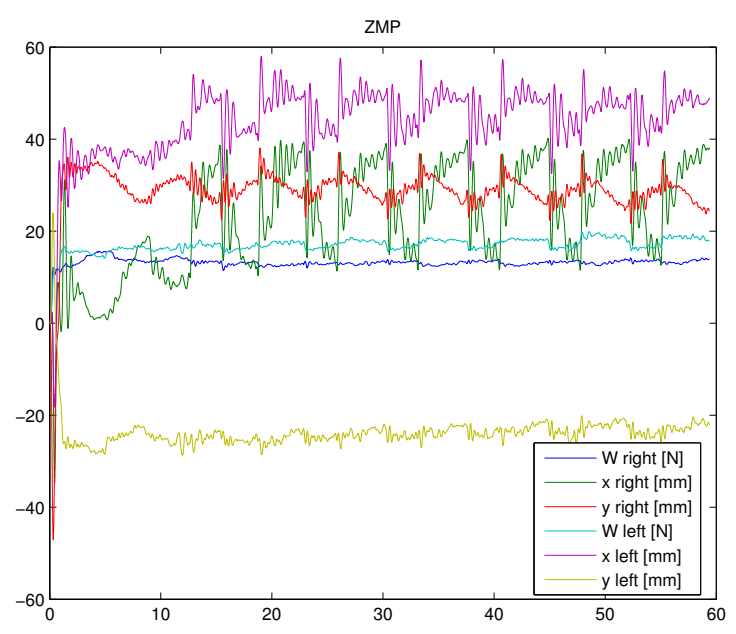

Fig. 10: ZMP measurements.

In Fig. 10, $W$ left and $W$ right are the amplitudes of the measured force for the left and right foot respectively, $x$ and $y$ frontal and lateral positions of the ZMP relative to the center of each foot.

According to the obtained results, one can observe that the produced motions are continuous and smooth, without switching phases, and the robot produces the desired squatlike motion (cf. Fig. 8).

\section{CONCLUSION AND FUTURE WORK}

This paper deals with a control scheme for humanoid robots. The proposed control solution is based on three tasks controlling (i) the relative distance between feet of the robot, (ii) the trajectory of its CoM with a ZMP regulation and (iii) the avoidance of joints' limits. One of the main advantages of such proposed control scheme lies in that fact that it provides a continuous control framework for wholebody motions. Indeed, the proposed principle don't use any decomposition of the movement in different phases, which avoid eventual discontinuities. The obtained results are very promising.

In future work, we aim at using different scenarios such as walking or following several objectives including for instance carrying or manipulating objects while walking.

\section{ACKNOWLEDGMENT}

This research was supported by the French National Research Agency, within the project R2A2 (ANR-09-SEGI-011).

\section{REFERENCES}

[1] S. Kajita, F. Kanehiro, K. Kaneko, K. Fujiwara, K. Harada, K. Yokoi, and $\mathrm{H}$. Hirukawa, "Biped walking pattern generation by using preview control of zero-moment point," in Proceedings of IEEE International Conference on Robotics and Automation (ICRA'03)., vol. 2, 2003, pp. $1620-1626$.
[2] T. Asfour, P. Azad, N. Vahrenkamp, K. Regenstein, A. Bierbaum, K. Welke, J. Schroeder, and R. Dillmann, "Toward humanoid manipulation in human-centred environments," Robotics and Autonomous Systems, vol. 56, no. 1, pp. 54-65, 2008.

[3] S. Nakaoka, A. Nakazawa, K. Yokoi, H. Hirukawa, and K. Ikeuchi, "Generating whole body motions for a biped humanoid robot from captured human dances," in Proceedings of IEEE International Conference on Robotics and Automation (ICRA'03)., vol. 3, 2003, pp. 3905-3910.

[4] S. Lengagne, N. Ramdani, and P. Fraisse, "Guaranteed computation of constraints for safe path planning," in Proceedings of IEEE International Conference on Humanoid Robots(Humanoids'07), 2007, pp. 312-317.

[5] J. Salini, S. Barthélemy, and P. Bidaud, "Lqp controller design for generic whole body motion," Climbing and Walking Robots and the Support Technologies for Mobile Machines, 2009.

[6] S. Lengagne, N. Ramdani, and P. Fraisse, "Planning and fast replanning safe motions for humanoid robots," IEEE Transactions on Robotics, vol. 27, no. 6, pp. 1095-1106, 2011.

[7] F.-J. Montecillo-Puente, M. N. Sreenivasa, and J.-P. Laumond, "On realtime whole-body human to humanoid motion transfer," in Proceedings of International Conference on Informatics in Control, Automation and Robotics (ICINCO'10), 2010, pp. 22-31.

[8] S. Kim, C. Kim, B. You, and S. Oh, "Stable whole-body motion generation for humanoid robots to imitate human motions," in Proceedings of IEEE International Conference on Intelligent Robots and Systems (IROS'09)., 2009, pp. 2518-2524.

[9] N. S. Pollard, J. K. Hodgins, M. J. Riley, and C. G. Atkeson, “Adapting human motion for the control of a humanoid robot," in Proceedings of IEEE International Conference on Robotics and Automation (ICRA'02)., vol. 2, 2002, pp. 1390-1397.

[10] Y. Nakamura, H. Hanafusa, and T. Yoshikawa, "Task-priority based redundancy control of robot manipulators," The International Journal of Robotics Research, vol. 6, no. 2, pp. 3-15, 1987.

[11] B. Siciliano and J. Slotine, "A general framework for managing multiple tasks in highly redundant robotic systems," in Proceedings of IEEE International Conference on Advanced Robotics (ICAR'91). 'Robots in Unstructured Environments', 1991, pp. 1211-1216.

[12] O. E. Ramos, L. Saab, S. Hak, and N. Mansard, "Dynamic motion capture and edition using a stack of tasks," in Humanoids, 2011, pp. 224-230.

[13] N. Mansard, O. Stasse, P. Evrard, and A. Kheddar, "A versatile generalized inverted kinematics implementation for collaborative working humanoid robots: The stack of tasks," in Proceedings of IEEE International Conference on Advanced Robotics (ICAR'09)., 2009, pp. 1-6.

[14] L. Sentis and O. Khatib, "A whole-body control framework for humanoids operating in human environments," in Proceedings of IEEE International Conference on Robotics and Automation (ICRA'06)., 2006, pp. 2641-2648.

[15] N. Mansard, O. Khatib, and A. Kheddar, "A unified approach to integrate unilateral constraints in the stack of tasks," IEEE Transactions on Robotics, vol. 25, no. 3, pp. 670-685, 2009.

[16] D. Galdeano, V. Bonnet, M. Bennehar, P. Fraisse, A. Chemori et al., "Partial human data in design of human-like walking control in humanoid robotics," in 10th International IFAC Symposium on Robot Control (SYROCO'12), Dubrovnik - Croatia, 2012, pp. 485-490.

[17] S. Kajita, H. Hirukawa, K. Harada, and K. Yokoi, Introduction à la commande des robots humanoides. Translated in French by Sakka, S. Springer, 2009.

[18] Z. Li, S. S. Sastry, and R. Murray, "A mathematical introduction to robotic manipulation," 1994.

[19] S. Cotton, P. Fraisse, and A. P. Murray, "On the manipulability of the center of mass of humanoid robots: Application to design," ASME Conference Proceedings, no. 44106, pp. 1259-1267, 2010.

[20] L. Sentis, J. Park, and O. Khatib, "Compliant control of multicontact and center-of-mass behaviors in humanoid robots," IEEE Transactions on Robotics, vol. 26, no. 3, pp. 483-501, 2010. 